\title{
Total Absorption Spectroscopy of Fission Fragments Relevant for Reactor Antineutrino Spectra and Decay Heat Calculations
}

\author{
A. Porta ${ }^{1, a}$, A.-A. Zakari-Issoufou ${ }^{1}$, M. Fallot ${ }^{1}$, A. Algora ${ }^{2,3}$, J.L. Tain ${ }^{2}$, E. Valencia ${ }^{2}$, S. Rice ${ }^{4}$, V.M. \\ Bui $^{1}$, S. Cormon ${ }^{1}$, M. Estienne ${ }^{1}$, J. Agramunt ${ }^{2}$, J. Äystö ${ }^{5}$, M. Bowry ${ }^{4}$, J.A. Briz ${ }^{1}$, R. Caballero- \\ Folch $^{6}$, D. Cano-Ott ${ }^{7}$, A. Cucouanes ${ }^{1}$, V.-V. Elomaa ${ }^{8}$, T. Eronen ${ }^{8}$, E. Estévez ${ }^{2}$, G.F. Farrelly ${ }^{4}$, \\ A.R. Garcia ${ }^{7}$, W. Gelletly,4, M.B Gomez-Hornillos ${ }^{6}$, V. Gorlychev6, J. Hakala ${ }^{8}$, A. Jokinen ${ }^{8}$, M.D. \\ Jordan $^{2}$, A. Kankainen ${ }^{8}$, P. Karvonen ${ }^{8}$, V.S. Kolhinen ${ }^{8}$, F.G. Kondev ${ }^{9}$, T. Martinez ${ }^{7}$, E. Mendoza ${ }^{7}$, \\ F. Molina ${ }^{2,10}$, I. Moore ${ }^{8}$, A. B. Perez-Cerdán ${ }^{2}$, Zs. Podolyák ${ }^{4}$, H. Penttilä8, P.H. Regan ${ }^{4,11}$, M. \\ Reponen $^{8,12}$, J. Rissanen ${ }^{8}$, B. Rubio ${ }^{2}$, T. Shiba ${ }^{1}$, A.A. Sonzogni ${ }^{13}$, C. Weber ${ }^{8,14}$, and IGISOL \\ Collaboration ${ }^{8}$
}

${ }^{1}$ SUBATECH, CNRS/IN2P3, Université de Nantes, Ecole des Mines de Nantes, F-44307 Nantes, France

${ }^{2}$ Instituto de Fisica Corpuscular (CSIC-Universitat de Valencia), Apartado Correos 22085, E-46071 Valencia,

Spain

${ }^{3}$ Institute of Nuclear Research, MTA ATOMKI, Debrecen, 4026 Hungary

${ }^{4}$ Department of Physics, University of Surrey, Guildford GU27XH, United Kingdom

${ }^{5} \mathrm{Helsinki}$ Institute of Physics, University of Helsinki, Fl-00014 Helsinki, Finland

${ }^{6}$ Universitat Politécnica de Catalunya (UPC), 08034 Barcelona, Spain

${ }^{7}$ Centro de Investigaciones Energéticas Medioambientales Y Tecnológicas, E-28040 Madrid, Spain

${ }^{8}$ Department of Physics, University of Jyväskylä, P.O. Box 35, Fl-40014 Jyväskylä, Finland

${ }^{9}$ Argonne National Laboratory, Argonne, IL 60439, USA

${ }^{10}$ Present address: Comisión Chilena de Energía Nuclear, Post Office Box 188-D, Santiago, Chile

${ }^{11}$ National Physical Laboratory, Teddington, Middlesex, TW11 OLW, United Kingdom

${ }^{12}$ Present address: RIKEN, 2-1 Hirosawa, Wako, Saitama 351-0198, Japan

${ }^{13}$ National Nuclear Data Center, Brookhaven National Laboratory, Upton NY 11973-5000, USA

${ }^{14}$ Present address: Faculty of Physics, Ludwig-Maximilians University Munich, Am Coulombwall 1, D-85748 Garching, Germany

\begin{abstract}
Beta decay of fission products is at the origin of decay heat and antineutrino emission in nuclear reactors. Decay heat represents about $7 \%$ of the reactor power during operation and strongly impacts reactor safety. Reactor antineutrino detection is used in several fundamental neutrino physics experiments and it can also be used for reactor monitoring and non-proliferation purposes. ${ }^{92,93} \mathrm{Rb}$ are two fission products of importance in reactor antineutrino spectra and decay heat, but their $\beta$-decay properties are not well known. New measurements of ${ }^{92,93} \mathrm{Rb} \beta$-decay properties have been performed at the IGISOL facility (Jyväskylä, Finland) using Total Absorption Spectroscopy (TAS). TAS is complementary to techniques based on Germanium detectors. It implies the use of a calorimeter to measure the total gamma intensity de-exciting each level in the daughter nucleus providing a direct measurement of the beta feeding. In these proceedings we present preliminary results for ${ }^{93} \mathrm{Rb}$, our measured beta feedings for ${ }^{92} \mathrm{Rb}$ and we show the impact of these results on reactor antineutrino spectra and decay heat calculations.
\end{abstract}

\footnotetext{
ae-mail: porta@subatech.in2p3.fr
} 


\section{Introduction}

In reactor cores, beta decay of fission products is at the origin of the emission of a large amount of electron antineutrinos. About $10^{21}$ neutrinos per second are emitted from a $1 \mathrm{GW}_{e l}$ power reactor. This makes reactors a powerful source for neutrino oscillation experiments and suggests the possibility of using this massive flux for applied purposes such as fuel or reactor monitoring. Indeed the reactor antineutrino flux depends on fuel composition and reactor power [1,2]. Motivated by an interest shown by the International Atomic Energy Agency in this idea [3], the scientific community started to actively explore this possibility with several dedicated experiments [4].

The beta decay of fission products is also at the origin of the decay heat which is about $7 \%$ of the reactor power during operation and the only source of energy when the reactor stops, generating strong safety constraints.

Both antineutrino spectra [5, 6]) and decay heat [7] can be calculated using the summation method which consists in summing the contribution to the desired quantity (decay heat or antineutrino energy) from each fission product present in the reactor core at a certain moment. In both fields, the advantage of this calculation respect to integral measurements is its usefulness in making predictions for future reactors. This is also an essential feature for safety and non-proliferation applications.

This method uses present nuclear data bases as input data for fission yields and decay data (energy spectra). Thus the precision of these data bases is extremely important for these calculations. One known problem affecting beta decay data bases is the so called "Pandemonium effect" [8], which arises from the difficulty that can occur in constructing nuclear level patterns for complex decays using Germanium detectors, especially when transitions are of high-energy or in regions of high level density, leading to a distortion in the reconstructed beta decay feeding.

The decays of ${ }^{92,93} \mathrm{Rb}$ are not well known in the data bases and they are candidates for the "Pandemonium effect". They have been selected [9] because they are major contributors to the antineutrino spectra. Moreover ${ }^{92} \mathrm{Rb}$ is indicated as priority 2 in decay heat calculations for the U/Pu cycle and priority 1 for the $\mathrm{Th} / \mathrm{U}$ cycle $[10,11]$.

In this paper we will present new preliminary results for the beta decay properties of ${ }^{92,93} \mathrm{Rb}$ obtained with TAS measurements and we will discuss the impact of these results on antineutrino spectra and decay heat calculations.

\section{Total Absorption Spectroscopy (TAS) measurements of ${ }^{92,93} \mathbf{R b}$}

A total absorption spectrometer should detect the de-excitation gammas emitted by the daughter nucleus after beta decay with an efficiency as close as possible to $100 \%$. The detection of the total gamma cascade allows for directly measuring the energy of the level fed and then the feeding probability can be deduced from the energy spectrum acquired during the experiment by solving the so called "inverse problem" $d=R \times f$, where $d$ is the measured energy spectrum, $R$ is the detector response and $f$ is the feeding to the excited levels in the daughter nucleus [12,13].

${ }^{92,93} \mathrm{Rb}$ have been measured at the IGISOL facility of the Jyväskylä University (Finland) [14] which is equipped with the double penning trap JYFLTRAP system [15], allowing for very precise selection of fission products. The TAS detector consisted of $12 \mathrm{BaF}_{2}$ crystals arranged in a compact geometry as shown in [16]. Each crystal is observed by a photomultiplier tube converting the scintillation light into an electrical signal proportional to the detected energy. The gamma detection efficiency is about $80 \%$ at $5 \mathrm{MeV}$. A beta detector has been placed in the centre of this spectrometer, facing the source 


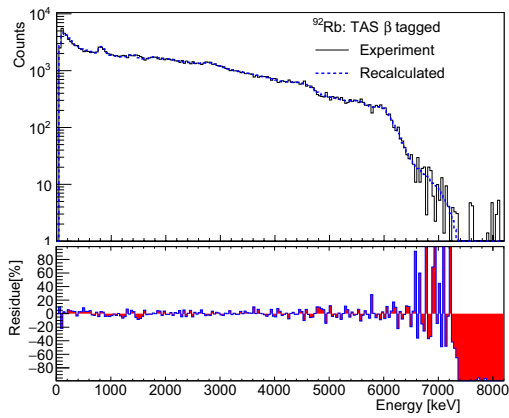

(a)

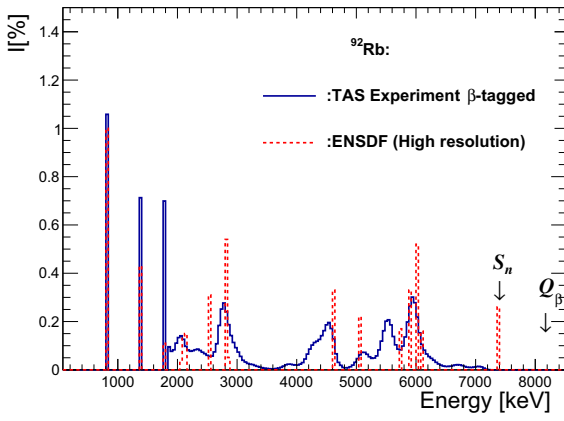

(b)

Figure 1: (a) Upper panel: Comparison between cleaned raw data (black continuous line) and reconstructed spectrum (blue dotted line) from beta feeding after solving the inverse problem for ${ }^{92} \mathrm{Rb}$. Lower panel: Residues between the two curves of the upper panel. (b) Comparison between the beta feeding from [9] in bleu continuous line and the feeding extracted from ENSDF data base [18] in red dotted line for ${ }^{92} \mathrm{Rb}$. The g.s. feeding of ${ }^{92} \mathrm{Rb}$ from [9] is $87.5 \pm 2.5 \%$ instead of the $95.2 \pm 0.7 \%$ reported in ENSDF. Figures are from [9].

implantation zone. This means that the background can be reduced by making coincidences between the beta event and the following gamma emission from the levels in the daughter.

\section{Data analysis}

Raw data from the experiment have been cleaned of contaminants. Thanks to the purity of the beam provided by the Jyväskylä facility and to the beta coincidence arrangement described before, the only contaminants were produced by the daughter nucleus decay and the pile-up. These contaminants have been subtracted as described in [9] for ${ }^{92} \mathrm{Rb}$. In the case of ${ }^{93} \mathrm{Rb}$, its daughter nucleus, ${ }^{93} \mathrm{Sr}$, has a neutron emission branch with $1.39 \%$ probability. Both gamma and neutron contaminants have been reproduced and subtracted as described in [17].

The resolution of the inverse problem to obtain the feeding distribution from these cleaned data has been performed using a maximisation expectation algorithm based on the Bayes theorem and combined with a $\chi^{2}$ minimisation [13]. For ${ }^{92} \mathrm{Rb}$, the spectrum obtained from the feeding estimated with the inverse problem resolution is shown in figure 1a in blue dotted line and compared with the clean experimental spectrum in black continuous line (figure from [9]). The lower panel of this figure shows the residues between the two curves. The same analysis procedure has been applied to ${ }^{93} \mathrm{Rb}$ as shown in [17].

\section{Results and impact on the antineutrino spectrum and decay heat calculations}

Figure $1 \mathrm{~b}$ shows the comparison between the beta feeding calculated as described in the previous section (blue continuous line) and the feeding extracted from the ENSDF data base [18] (red dotted 


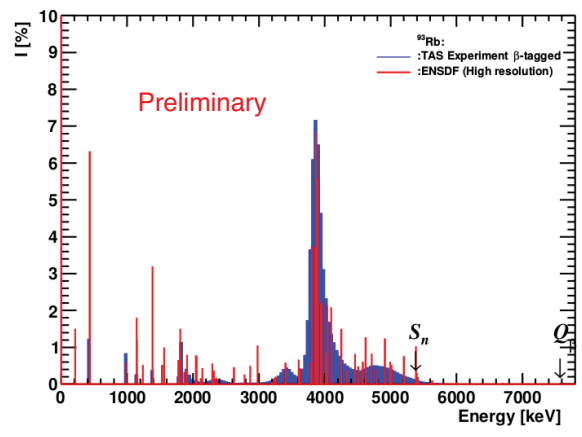

Figure 2: Comparison between the beta feeding from [17] in blue and the feeding extracted from ENSDF data base [18] in red for ${ }^{93} \mathrm{Rb}$.

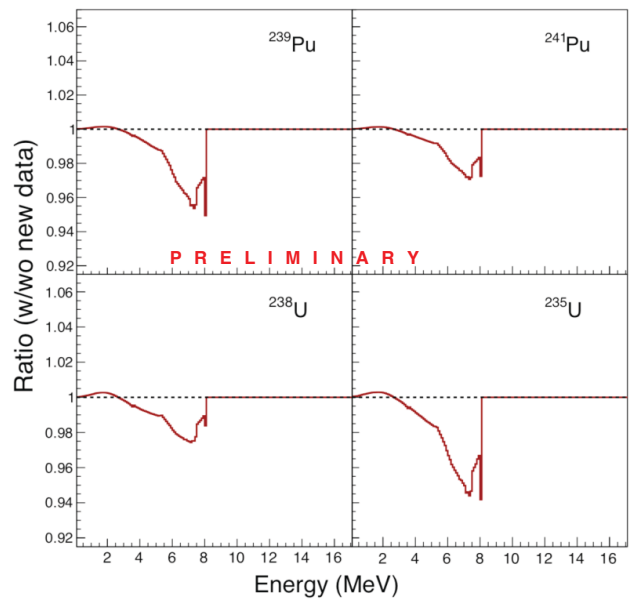

Figure 3: Ratio between antineutrino spectra for ${ }^{235,238} \mathrm{U}$ and ${ }^{239,241} \mathrm{Pu}$ calculated with the new TAS data for ${ }^{92,93} \mathrm{Rb}$ and with previous data from Rudstam et al. [20].

line) for ${ }^{92} \mathrm{Rb}$ (figure from [9]). The ground state (g.s.) feeding of ${ }^{92} \mathrm{Rb}$ from this work is $87.5 \pm 2.5$ $\%$ instead of the $95.2 \pm 0.7 \%$ reported in ENSDF.

Preliminary results for ${ }^{93} \mathrm{Rb}$ are shown in figure 2 and [17]. The results of this analysis (blue) are in quite good agreement with the data from ENSDF data base (red) that already contains results from previous TAS measurements for this nucleus from Greenwood et al. [19].

Figure 3 contains the ratio between the antineutrino spectra calculated with and without these new data and emitted by ${ }^{235,238} \mathrm{U}$ and ${ }^{239,241} \mathrm{Pu}$ isotopes. The spectra without these data are calculated as described in [6] using Rudstam's [20] results for ${ }^{92,93} \mathrm{Rb}$. More details about the calculation of the spectra can be found in [9].

From figure 3 we can see that, as predicted in [9], the main impact of these data is in the 4-8 MeV region, with a maximum variation around $7-8 \mathrm{MeV}$ of $6 \%$ for ${ }^{235} \mathrm{U}, 5 \%$ for ${ }^{239} \mathrm{Pu}, 3 \%$ for ${ }^{241} \mathrm{Pu}$ and 
$2.5 \%$ for ${ }^{238} \mathrm{U}$.

The impact of these two nuclei shown in figure 3 underlines the importance of TAS measurements if we want to improve the calculation of reactor antineutrino spectra through the summation method. Regarding decay heat calculations, our measurements give a mean energy for the emitted gamma spectrum of $464.2 \mathrm{keV}$ and for the emitted beta spectrum of $3.50 \mathrm{MeV}$. Mean values from ENDF7.1.1 (g.s. to g.s. feeding $95.2 \%$ ) are $170 \mathrm{keV}$ for the mean gamma energy and $3.63 \mathrm{MeV}$ for mean beta energy [21]. This is mainly due to the change of g.s. to g.s. feeding from $95.2 \%$ to $87.5 \%$ and goes in the direction of increasing the decay heat component released by gammas and reducing the component due to beta particles. This nucleus is important for ${ }^{235} \mathrm{U}$, for which summation calculations are less in agreement with Tobias' compilation [22] as shown in [23].

\section{References}

[1] A. Bernstein et al., J. Appl. Phys. 103, 074905 (2008)

[2] Yu.V.Klimov et. al., Atomic Energy, 76, 123 (1994)

[3] IAEA Report STR-361, Department of Safeguards. Proceedings of the first meeting of the Ad Hoc Working Group on Safeguards Applications utilising Antineutrino Detection and Monitoring, IAEA Report SG-EQGNRL-RP-0002 (2012).

[4] Applied Antineutrino Workshop 2015, presentations at: https://indico.in2p3.fr/event/10050/otherview? view $=$ standard

[5] Th. A. Mueller et al., Phys.Rev. C 83, 054615 (2011)

[6] M. Fallot et al., Phys. Rev. Lett. 109 , 202504 (2012)

[7] T. Yoshida et al., J. Nucl. Sci. Technol. 36, 135(1999).

[8] J.C. Hardy et al., Phyics Letter B 71, 307 (1977)

[9] A.-A. Zakari-Issoufou et al., Phys. Rev. Lett. 115, 102503 (2015)

[10] Assessment of Fission Product Decay Data for Decay Heat Calculations , Nuclear Science NEA/WPEC-25 (2007).

[11] Decay Heat Calculation: Assessment of Fission Product Decay Data Requirements for $\mathrm{Th} / \mathrm{U}$ Fuel, INDC(NDS)-0577

[12] D. Jordan, A. Algora, et al., Phys. Rev. C 87, 044318 (2013).

[13] J. L. Tain et al., Nucl. Instrum. Methods. Phys. Res.Sect. A 571, 728 (2012).

[14] I. D. Moore et al., Hyperfine Interact. 223, 17 (2014).

[15] T. Eronen et al., Eur. Phys. J. A 48, 46 (2012).

[16] A. Algora et al., Nucl. Data Sheets 120, 12 (2014).

[17] A.-A. Zakari, Ph.D. thesis, Universit Nantes, France (2015).

[18] C. Baglin, Nucl. Data Sheets 113, 2187 (2012)

[19] R. C. Greenwood, R. G. Helmer, M. H. Putnam, and K. Watt, Nucl. Instrum. and Methods Phys. Res. Sect. A 390, 95 (1997).

[20] G. Rudstam et al., At. Data and Nucl. Data Tables 45, 239 (1990).

[21] A. Sonzogni private communication.

[22] A. Tobias, Decay Heat, Prog. Nucl. Energy 51 (1980).

[23] Total Absorption Gamma-ray Spectroscopy for Decay Heat Calculations and Other Applications, INDC(NDS)-0676 
